\title{
Detecting deceptive communication through linguistic concreteness
}

\author{
Bennett Kleinberg ${ }^{1,2}$, Isabelle van der Vegt ${ }^{1}$, Arnoud Arntz ${ }^{2}$, Bruno Verschuere ${ }^{2}$ \\ ${ }^{1}$ Department of Security and Crime Science, University College London, UK \\ ${ }^{2}$ Department of Psychology, University of Amsterdam, The Netherlands
}

\begin{abstract}
Several research lines attempted to tell truthful from deceptive texts by looking at the concreteness in language as an indicator of truthfulness. We identified eight different operationalizations of concreteness for computerautomated analysis and validated these operationalizations on six diverse datasets containing truthful and deceptive texts (about hotel reviews, past and future weekend plans, as well as intended flight plans). The results suggest that not just the efficacy but also the directionality of concreteness as a cue to deception is dependent on the operationalization and the context. A predictive analysis suggests that concreteness indices can differentiate between truthful and deceptive statements above the chance level and are a worthy path for future research. The importance of conducting multi-dataset and multi-operationalization investigations in linguistic deception detection research is highlighted.
\end{abstract}

Transparency statement:

We report all data exclusions, all manipulations, and all measures in the study. The data, materials, and code to reproduce the analysis are publicly available on the Open Science Framework at https://osf.io/uyrb5. 


\section{Introduction}

\section{Concreteness in deception detection}

In recent years, deceptive language has received considerable attention in the field of computational linguistics. Numerous approaches aim to discriminate between truthful and deceptive texts, for example by automatically scoring texts on their richness of detail (Bond et al., 2017; Bond \& Lee, 2005) or the proportion of named entities (Kleinberg, Mozes, Arntz, \& Verschuere, 2017). Little is known about whether these approaches can be integrated through a common, underlying feature that may explain why a text is classified as truthful or deceptive. In the current paper, we explore the use of the construct of concreteness as a general feature for the discrimination between truthful and deceptive texts. Concreteness is defined as the 'degree to which the concept denoted by a word refers to a perceptible entity' (Brysbaert, Warriner, \& Kuperman, 2014, p. 904). Notably, the level of concreteness itself has not yet been explicitly implemented as a feature for linguistics-based deception detection. However, we suspect that measures used in previous research such as specificity (Burgoon \& Qin, 2006), the richness of detail (Bond \& Lee, 2005), and information specificity (Kleinberg, Mozes, et al., 2017) may have served as proxies of concreteness instead. Drawing on theories of verbal deception detection, we explain why the construct may be of particular importance to recognize deceptive and truthful language. After that, we compare eight different operationalizations of concreteness on six datasets of truthful and deceptive texts. From this investigation, we conclude the suitability of concreteness for automatic classification of truthful and deceptive texts, in addition to discussing the theoretical implications of our findings for the field in general.

\section{Theoretical rationales for using concreteness for deception detection}

Four theories of deception detection, when viewed together, suggest that concreteness may be of importance. The first is Reality Monitoring (Johnson, Bush, \& Mitchell, 1998), which indicates that truthful memories are obtained through sensory experiences and deceptive memories are constructed through cognitive operations. Therefore, it is expected that truthful descriptions contain more emotional, perceptual, spatial and temporal details than deceptive descriptions. Studies that have used these features to score texts have shown that truthful statements are indeed richer in detail (Masip, Sporer, Garrido, \& Herrero, 2005). Importantly, one could argue that descriptions of actual sensory experiences are more likely to be concrete than made-up descriptions arising from cognitive operations.

Second, Criteria-based Content Analysis (CBCA) assumes that truthful narratives are different from deceptive narratives in their quality and content (Undeutsch, 1989). Among several indicators of CBCA (e.g., logical structure, spontaneous corrections, descriptions of unexpected complications; see Köhnken, 2004), is the degree to which the narrative is embedded in a broader context. Contextual embedding refers to the notion that the description of an event is placed within the context of a specific place and time, as well as the extent to which the event is related to any relevant personal relationships. Indeed, it has been found that truthful accounts contain more contextual embeddings than fabricated accounts (Köhnken, 2004). Again, it can be argued that contextually embedding an event promotes and requires concrete language use.

The third theoretical line, the Verifiability Approach (Nahari, Vrij, \& Fisher, 2014), explains that a liar would be inclined to be detailed in his/her statement, yet has to avoid giving information that can be verified. That is, liars are confronted with a dilemma; they are aware that their narrative needs to be detailed to be believed, but 
they risk being incriminated if specific, testable information they give turns out to be false when checked. For instance, 'I'm meeting Sarah at Starbucks' would be considered verifiable information, whereas 'I'm meeting someone for a coffee later' would not. The number of verifiable details has been successfully used to discriminate between liars and truth tellers (e.g., Harvey, Vrij, Leal, Lafferty, \& Nahari, 2017; Jupe, Leal, Vrij, \& Nahari, 2017). In this line of research, a verifiable detail is considered to be any activity that has been i) done with an identifiable person, ii) has been witnessed by an identifiable person, or iii) has been recorded by technology. In essence, concrete information about specific persons or locations are a vital part of verifiability, and the lack thereof can serve as a linguistic feature of deception.

The fourth theoretical line, known as Construal Level Theory (CLT), describes how people represent a situation in the future or past as being close or far away from the self. That is, when an event is distant in time, is likely to happen to someone other than the self, or is unlikely to happen in the first place (i.e., hypothetical distance), the representation of that event will be more abstract (Trope \& Liberman, 2010). On the other hand, if a future event is psychologically close, it is more likely to be precisely planned out. Such planning typically occurs in the form of implementation intentions (Gollwitzer, 1999), which are "if ... then ..." statements about how, when, and where a goal can be realized. In terms of language, it has indeed been found that people speak in abstract "why" descriptions if an event is distant, but switch to specific "how" descriptions (reflecting implementation intentions) when an event becomes close in psychological distance (Geurts, Granhag, Ask, \& Vrij, 2016; Liberman \& Trope, 1998; Vallacher \& Wegner, 1987). Within the deception detection literature, it has been argued that the psychological distance of an event can be translated into verbal cues to deception. For instance, if a person is lying about fulfilling a future plan, the event is likely to be farther away regarding psychological distance than for a person who is genuinely planning to fulfil the plan. Only the latter has made implementation intentions. In other words, the genuine future plan (e.g., catching a flight) of a truth-teller is more likely to be concrete than that of a liar. Indeed, people who spoke the truth about their intentions have been found to speak more about "how", whereas those lying where more likely to speak about "why", providing justifications for their intentions instead (Granhag \& Mac Giolla, 2014).

In sum, the four theoretical rationales point toward a prevalent profile: truthful accounts are expected to be more specific, concrete and embedded within a situational context, whereas deceptive statements are expected to be more abstract and contain less concrete information. One way in which this can be explained is that liars are less able to recall concrete details of an event and - in the case of the Verifiability Approach - are reluctant to provide specific information. The predictions made by this view are henceforth referred to as the truthful concreteness hypothesis (more concrete language in truthful accounts). However, contradicting predictions stating that deceptive rather than truthful statements are more concrete - have also been made. Researchers hypothesized that deceptive narratives contain fewer markers of cognitive complexity than truthful ones, resulting in the deceptive language being more concrete (Newman, Pennebaker, Berry, \& Richards, 2003). Liars were expected to tell less complex stories than truth tellers because much of the cognitive resources are spent on the act of lying itself. Here the postulated mechanism is that the additional cognitive load involved in lying depletes resources resulting in liars' use of simpler and hence more concrete language than truth-tellers. The authors argue that liars will construct simple narratives with more motion verbs (e.g., walk, move, go) because simple, concrete actions represented by motion verbs are supposedly easier to describe than evaluations and judgments (e.g., "Usually I take the bus, but it was such a nice day"). Indeed, the authors found that liars across five independent 
samples used more motion verbs. Also, liars were found to use fewer third-person pronouns (e.g., she, her) than truth-tellers, and instead opted for the more specific nouns (e.g., a woman, my sister) and proper names when describing persons (Newman et al., 2003). Moreover, research into deceptive intentions found that liars can be overly specific compared to truth-tellers (Kleinberg, Toolen, Vrij, Arntz, \& Verschuere, 2018). Contrary to the view arising from RM, CBCA, the VA, and the CLT, these findings were interpreted concerning liars' overcompensation. The authors speculate that liars may have added unrealistically many concrete details to their narrative to appear truthful. We refer to the latter two dynamics as the deceptive concreteness hypothesis (more concrete language in deceptive accounts).

The precise directionality of concreteness concerning deception seems inconclusive. However, the several theoretical lines described thus far suggest that the concept of concreteness might be at play in truthful and deceptive language. To date, an explicit and comprehensive investigation into concreteness as a cue to deception is lacking. With the current paper, we aim to address this matter.

\section{Context}

Besides the mixed predictions that stem from the four theoretical rationales discussed thus far, context is an additional factor that impacts on linguistic indicators of deception and may likely do so on the concept of concreteness as a cue. In communication literature, contextual influences on interpersonal communication are widely recognized, such as the physical setting of an interaction, the social setting (i.e. the relationship between communicators), the institutional setting, the functional goal of the interaction, and the cultural setting in which an interaction takes place (see Burleson, 2009). Indeed, a large body of work has shown that some of these contexts also have an influence on linguistic cues to deception, such as the communication modality and the degree to which an individual is motivated to deceive (Qin, Burgoon, Blair, \& Nunamaker, 2005). Linguistic cues also depend on the type of the deception that is studied (e.g. concealment, fabrication, or equivocation, Buller, Burgoon, Buslig, \& Roiger, 1994, 1996). A meta-analysis examining computer-based deception detection showed that linguistic cues to deception were moderated by event type, personal involvement, emotional valence, intensity of interaction, and sender motivation (Hauch, Blandón-Gitlin, Masip, \& Sporer, 2015). The authors stress that deception researchers should strive for experiments that mirror real life deception, for example by allowing for preparation and inducing high levels of motivation. But even in experiments were such requirements are met, other contextual factors that influence deception cues remain, such as event and interaction types. Therefore, it is important to understand which cues to deception operate in which contexts, including the concept of concreteness. Not only do different theories of deception argue for different directionalities for concreteness, the potential cue may be further impacted by the context in which deception is studied.

\section{Linguistic indices of concreteness}

Even though concreteness has yet to be explicitly tested as a cue to deceit, concreteness is not a new construct in computational linguistics. We discuss three operationalizations of concreteness, which later will be of importance to our investigation into the role of the construct in deception detection. (1) The linguistic category model (LCM) is used to assess the overall concreteness of texts. Within the LCM, five categories of adjectives and verbs are placed on a continuum ranging from concrete to abstract (Semin \& Fiedler, 1988). Descriptive action verbs (DAV; e.g., walk, catch, touch) are considered the most concrete, interpretative action verbs (IAV; e.g., help, cheat, 
imitate) are considered less concrete, and finally, state verbs (SV; e.g., admire, fear, desire) are considered the least concrete. Adjectives are considered more abstract than verbs (e.g., charming, peaceful, jealous), and finally, nouns (e.g., athlete, teacher, father) are considered the most abstract (see also the motion verb argument by Newman et al., 2003). The LCM has been used to assess the concreteness-abstractness continuum of texts written from first-person and third-person perspectives (Seih, Beier, \& Pennebaker, 2017). Using the LCM algorithm, the authors found texts written in third-person to be higher in levels of abstraction than those written in the firstperson perspective. A similar effect may be expected in truthful versus deceptive accounts, where truthful accounts are experienced first-person by the sender and therefore may include more concrete information and less abstraction.

(2) A different operationalization of concreteness is the sum of articles (e.g., a, an, the), prepositions (e.g., at, on, in), and quantifiers (e.g., a lot, many) in a text. These words signal concreteness because concrete words 'are associated with more contextualised and detailed representations of objects' (Larrimore, Jiang, Larrimore, Markowitz, \& Gorski, 2011, p. 23). Because articles refer to nouns, prepositions specify relationships between objects and people, and quantifiers express degrees of difference between objects, their function may be considered in line with the definition above of concreteness. Based on these assumptions, the inverse of the sum of the proportion of articles, prepositions, and quantifiers has been used as an abstraction index for fraudulent scientific papers (Markowitz \& Hancock, 2016). The authors found that fraudulent scientific papers were written more abstractly (i.e., contained fewer articles, prepositions, and quantifiers) than non-retracted papers. Within the context of the present paper, one could similarly expect deceivers to provide less concrete language, thus fewer articles, prepositions, and quantifiers.

(3) Brysbaert et al. (2014) asked people to rate the degree to which they perceived certain words as concrete, resulting in a dataset of concreteness ratings for almost 40,000 words based on the ratings from 4,000 participants. Human judges were instructed to rate each word on a scale from 1 to 5 , with $1=\operatorname{abstract}$ and $5=$ concrete, resulting in a direct measure of concreteness and abstraction. Abstract words were defined as referring to a meaning that cannot be experienced directly (i.e. the meaning of the word can only be defined by other words). Concrete words were defined as those that refer to something in reality, which can be experienced through one of the five senses. If this concept is used for the purpose of deception detection, it could be argued that truthful accounts indeed refer more to concepts or events in reality.

\section{Concreteness in deception}

Some investigations into truthful and deceptive language have examined constructs that resemble concreteness. That is, the measures discussed hereafter may have served as potential proxies for concreteness within the study of deception detection. The idea of Reality Monitoring has been extended, for instance, by including first-person pronouns, second person pronouns, and third-person pronouns in addition to sensory details (Zhou, Burgoon, Nunamaker, \& Twitchell, 2004). These linguistic properties were described as factors as constituting a measure of specificity as 'language that establishes the context of the statement and perceptual information given' (Fuller, 2008, p. 60), a definition that arguably also holds for concreteness. The resulting framework (termed the BurgoonZhou framework) was used in automated analysis of truthful and deceptive language, using pronouns (first, second and third) and perceptual processes (seeing, hearing, feeling) to represent the construct of specificity. Perceptual information is likely to refer to concepts in reality, which closely resembles the Brysbaert et al. (2014) definition 
of concreteness, where highly concrete words are considered to refer to concepts in reality that can be experienced through the senses.

Another automated measure of deception that closely resembles concreteness focuses on named entity recognition (Kleinberg, Mozes, et al., 2017). The latter method extracts single words or phrases from a text and classifies them as, for example, specific person names, organizations and times (e.g., "Sarah," "Starbucks," "3:00 PM"). The specificity of named entities might serve as a means to automatically capture the nature of verifiable details (e.g., specific names of people or places). Liars, therefore, may be inclined to avoid mentioning such named entities. The proportion of named entities was - as expected - higher in truthful than in deceptive statements. In a similar vein, a case can be made for the concreteness of specific words as a feature of deception. Specific names of persons, organizations, and locations would typically refer to a concrete, tangible, or at least perceptible entity (e.g., "Sarah," "Starbucks," "New York").

Table 1. Linguistic operationalizations of concreteness.

\begin{tabular}{|c|c|}
\hline Operationalization & Details \\
\hline Concreteness (Brysbaert et al., 2014) & $\begin{array}{l}\text { Human ratings of abstract }(1=\text { meaning cannot be } \\
\text { experienced directly) to concrete }(5=\text { refer to something in } \\
\text { reality) }\end{array}$ \\
\hline Linguistic Category Model (Seih et al., 2017) & $\begin{array}{l}\text { Scale ranging from concrete to abstract: descriptive action } \\
\text { verbs, interpretative action verbs, state verbs, adjectives, } \\
\text { nouns. }\end{array}$ \\
\hline Concreteness index (Markowitz \& Hancock, 2016) & $\begin{array}{l}\text { The inverse of the sum of the proportion of articles, } \\
\text { prepositions, and quantifiers. }\end{array}$ \\
\hline Named entities (Kleinberg, Mozes, et al., 2017) & $\begin{array}{l}\text { Classifying words or phrases into, e.g. specific names, } \\
\text { organizations or times }\end{array}$ \\
\hline Reality Monitoring (Bond et al., 2017; Bond \& Lee, 2005) & $\begin{array}{l}\text { Increased perceptual, spatial and temporal details, } \\
\text { decreased cognitive operations }\end{array}$ \\
\hline Linguistic specificity (Zhou et al., 2004) & $\begin{array}{l}\text { Increased perceptual details, first person pronouns, second } \\
\text { person pronouns, third person pronouns }\end{array}$ \\
\hline Person index & $\begin{array}{l}\text { Personal pronouns, proper nouns and person names } \\
\text { (ranging from abstract to concrete) }\end{array}$ \\
\hline Motion verbs (Newman et al., 2003) & $\begin{array}{l}\text { Concrete, simple motion verbs (i.e. concrete) versus } \\
\text { complex evaluations and judgments (i.e. abstract) }\end{array}$ \\
\hline
\end{tabular}

The current study

Reality Monitoring, Criteria-based Content Analysis, the Verifiability Approach and the Construal Level Theory predict truth-tellers to use more concrete language than liars. On the other hand, liars have also been found to be more concrete than truth-tellers in linguistic analyses using the LIWC (Newman et al., 2003). We translated these theoretical insights and empirical findings into two hypotheses: i) the truthful concreteness hypothesis: truthteller's language is more concrete than that of liars because liars fail to recall an event in sufficient detail from memory. Alternatively, we propose ii) the deceptive concreteness hypothesis: liars' language is more concrete than that of truth-tellers because they are motivated to appear convincing and attempt to do so by incorporating specific, concrete information into their narratives.

Reviewing the literature from various fields (computational linguistics, legal psychology, cognitive psychology) we identified eight ways to operationalize concreteness: 1) crowd-sourced concreteness ratings (Brysbaert et al., 2014), 2) the Linguistic Category Model (Seih et al., 2017; Semin \& Fiedler, 1988), 3) the 
Abstraction Index (Markowitz \& Hancock, 2016), 4) the proportion of named entities (Kleinberg, Mozes, et al., 2017), 5) motion verbs (derived from Newman et al., 2003), 6) a Reality Monitoring score (Bond et al., 2017; Bond \& Lee, 2005), 7) the Burgoon-Zhou model of specificity (Fuller, 2008; Zhou et al., 2004), and 8) a new person reference index (see Table 1). There is a reason to suspect that these eight operationalizations may not all function in the same way in the context of verbal deception detection. For instance, some authors found no difference between truths and lies (e.g., "specificity" in Zhou et al., 2004), others found more concrete information in truths than in lies (e.g., "RM" in Bond et al., 2017; named entities in Kleinberg, Mozes, et al., 2017), and others found lies to be more concrete than truths (Newman et al., 2003). We systematically assessed their validity as a cue to deception on six different datasets of truthful and deceptive texts.

\section{Method}

\section{Datasets}

We used six datasets in the current study (Table 2). The first two datasets consist of positive (Ott, Choi, Cardie, \& Hancock, 2011) and negative (Ott, Cardie, \& Hancock, 2013) hotel reviews that were either genuine (i.e., verified and obtained from hotel review websites) or fabricated by participants. Further, we used typed statements where participants either told the truth or lied about what they did on the past weekend, as well as data from two experiments on genuinely planned, future weekend activities (truthful) and fabricated future weekend activities (Kleinberg et al., 2018), and a dataset containing truthful and deceptive statements about participants' planned flights (Kleinberg, Nahari, Arntz, \& Verschuere, 2017). The hotel reviews (2x) and the past weekend activities pertain to truthful and deceptive statements about past experiences, while the future weekend plans $(2 \mathrm{x})$ and the statements about the upcoming flight are about future experiences.

Table 2. Summary of the datasets used.

\begin{tabular}{ll}
\hline \multicolumn{1}{c}{ Dataset } & \multicolumn{1}{c}{ Sample characteristics } \\
\hline & Past experiences \\
Positive hotel reviews & 400 truthful, positive reviews from Tripadvisor (2011), 400 deceptive positive reviews \\
written by users of Amazon Mechanical Turk & 400 truthful negative reviews (from Expedia, Hotels.com, Orbitz, Priceline, Tripadvisor, \\
Negative hotel reviews & $\begin{array}{l}\text { and Yelp), } 400 \text { deceptive negative reviews written by users of Amazon Mechanical Turk } \\
81 \text { truthful and } 67 \text { deceptive statements about an activity during the past weekend }\end{array}$ \\
Past activities & \multicolumn{1}{c}{ Future experiences } \\
Planned activities I & 86 truthful statements and 86 deceptive statements about planned activities on the \\
forthcoming weekend (Exp. 1) & 104 truthful statements and 101 deceptive statements about planned activities on the \\
forthcoming weekend (Exp. 2) & 109 truthful and 113 deceptive statements about an upcoming flight in the next 3 months \\
\hline
\end{tabular}


For the current study, we used eight different operationalizations previously published on the abstractionconcreteness dimension (see Table 1). The text processing, extraction of linguistic constructs and analyses was performed in $R$ (R Core Team, 2016) and the full analysis code and data are available at https://osf.io/uyrb5.

\section{Judged Concreteness}

To calculate the average concreteness per text statement, we used the word concreteness annotation dataset from Brysbaert et al. (2014). Specifically, we tokenized all texts and retrieved the average concreteness score for each token. Therefore, each token per text statement that was contained in the annotated concreteness data was assigned its corresponding concreteness score. The dependent variable "judged concreteness" is the average of the concreteness score of all tokens per text. Because the matching of words between the original text and the concreteness dataset is sensitive to word variations (e.g., "beard" vs. "bearded"), we also performed these steps on stemmed tokens (i.e. reducing each word to its core, e.g., "concrete" to "concret" and "oscillating" to "oscil") in the original texts and in the concreteness dataset. This procedure ensures a bigger overlap between words in participants' statements and those judged in Brysbaert et al.'s data. This led to the dependent variable "judged concreteness (stemmed)". Higher scores indicate more concrete language for "judged concreteness" and "judged concreteness (stemmed)".

\section{Linguistic Category Model (LCM) score}

We calculated the LCM score for each text according to the algorithm introduced by Seih et al. (2017). Accordingly, the LCM score consisted of the proportion of descriptive action verbs (DAV), interpretative action verbs (IAV), state verbs (SV), adjectives and nouns. To extract DAV, IAV and SV, we used the Harvard General Inquirer (http://www.wjh.harvard.edu/ inquirer/) and extracted the proportion of each verb category using $R$. The proportion of adjectives and nouns was extracted in $R$ using the Spacy-to-R bridge spacyr (Benoit \& Matsuo, 2017) that allows to use the Spacy natural language processing tool written in python (Honnibal, 2017) in R. The final LCM score consisted of the weighted average of these categories divided by the sum of the sub-indices, so that the final score fell between 1 (very concrete) and 5 (very abstract).

\section{Abstraction index score}

Following the abstraction index proposal (Markowitz \& Hancock, 2016), we used the inverse sum of articles, prepositions, and quantifiers to model the abstraction contained in each text. We used the 2015 version of the Linguistic Inquiry and Word Count (LIWC; Pennebaker, Boyd, Jordan, \& Blackburn, 2015) software to extract the proportion of articles, prepositions, and quantifiers. The LIWC is a psycholinguistic dictionary with categories that reflect linguistic variables, producing proportions of each lexical category for a given text.

\section{Named entities}

We extracted the proportion of named entities in each of 18 named entity categories (e.g., persons, times, dates, see https://spacy.io/usage/linguistic-features\#entity-types) using spacyr (Benoit \& Matsuo, 2017) This procedure was adopted from previous research (Kleinberg, Mozes, et al., 2017). High proportions of named entities serve to represent high levels of concreteness. 


\section{Reality Monitoring (RM) score}

Contrary to the traditional manual annotation of Reality Monitoring variables, Bond et al. (2017) proposed a computer-automated measurement of the RM richness of detail using the LIWC. Specifically, we calculated an RM index as suggested: perceptual processes (LIWC category "percept") + temporal details (LIWC category "time") + spatial details (LIWC category "space") - cognitive operations (LIWC category "cogproc"), with high scores on this composite measure representing high levels of concreteness.

\section{Burgoon-Zhou specificity}

We calculated an index score of what Fuller (2008) labelled the Burgoon-Zhou specificity. That score was the sum of the following sub-indices: first-person pronouns (LIWC categories "i" and "we"), second person pronouns (LIWC category "you"), third person pronouns (LIWC categories "shehe", "they"), modifiers (part-of-speech proportions of adjectives and adverbs), the proportion of sensory details (LIWC category "percept") and the number of sensory details (LIWC category "percept" multiplied by the number of words per statement). Again, high levels of this index score represent high levels of concreteness.

\section{Person reference index}

References to persons can be done on multiple levels in a statement (Newman et al., 2003). In its simplest form, a person reference is made through personal pronouns ('he,' 'they'). A more specific reference would include mentioning proper nouns that refer to persons ('friend,' 'daughter'). We extend previous work on this issue by adding a third, most specific person reference in the form of person named entities (e.g., 'Felix'). To derive a person index score, we assigned simple weights reflecting the magnitude of specificity in person references: 1 *personal pronouns (LIWC category "ppron") +2 *proper person nouns (LIWC category "social") $+3 *$ person named entities. We divided this score by the sum of the three sub-indices to obtain a score between 1 (low in person specificity, suggesting low concreteness) and 3 (high in person specificity, suggesting high concreteness).

\section{Motion verbs}

Newman et al. (2003) proposed a linguistic model that consists of self-references, other-references, exclusive words, negative emotions and motion verbs. Specifically, motion verbs (e.g., 'walk,' measured here with the LIWC category "motion") were hypothesized to be higher in deceptive than in truthful language. In line with the cognitive load theory of deception (Zuckerman, DePaulo, \& Rosenthal, 1981), it was argued that the liars' task of fabricating a believable account take up so many cognitive resources that they resort to simple, concrete language of describing actions, which is easily constructed with motion verbs. Here, concrete (simple) language is seen as indicative of deceptive language. Furthermore, Newman et al. (2003) present evidence that motion verbs were negatively correlated with cognitively complex language. Consequently, we would expect motion verbs to be more prevalent in deceptive statements than in truthful ones, representing high concreteness.

\section{Quantifying the truth-lie difference}

To measure the difference in the dependent variables between truthful and deceptive texts, we used the Cohen's $d$ (between-subjects) effect size (Cohen, 1988). As an indicator of the magnitude of the effects, Cohen suggested 
that effect sizes of $0.20,0.50$, and 0.80 should be considered small, moderate and large, respectively. We also conducted a mini-meta-analysis (Valentine, Pigott, \& Rothstein, 2010) per operationalization for all statements as well as for past and future experiences separately. The meta-analysis was conducted with the metaphor $\mathrm{R}$ package (Viechtbauer, 2010) using a random effects model with a restricted maximum-likelihood estimator. ${ }^{1}$

\section{Results}

Table 3 shows the findings for all operationalizations of the concreteness-abstraction dimension (see Appendix A for the means and standard deviations).

\section{Judged concreteness}

The average concreteness score was higher in truthful positive and negative hotel reviews than in deceptive ones ( $d=0.45$ and $d=0.52$, respectively), and higher in truthful statements about past activities than in deceptive ones $(d=0.49)$. There was no significant difference in the average concreteness score for any of the future experience datasets. For the stemmed version of the concreteness score, the effect sizes for the three past experience datasets remained relatively stable. Also, the stemming resulted in significantly higher concreteness scores for truthful than for deceptive statements about future weekend plans in two of the three datasets ( $d=0.39$ and $d=0.40$ ). The meta-analytical results support the hypothesis that truthful language is more concrete than deceptive language $\left(d_{\text {meta }}=0.41\right)$, an effect that is larger for past experiences $\left(d_{\text {meta }}=0.52\right)$ than for future experiences $\left(d_{\text {meta }}=0.28\right)$. These findings also suggest that the stemmed concreteness version is more suitable than the one based on the raw texts, most likely due to better matches between the concreteness database and the texts.

\section{Linguistic Category Model score}

The LCM score was larger for truthful hotel reviews than for deceptive ones suggesting that truthful reviews were more abstract than deceptive ones (positive reviews: $d=0.27$; negative reviews: $d=0.19$ ). There were no significant differences for the other datasets, and the meta-analysis indicated that overall truthful statements have a slightly higher LCM score than deceptive ones $\left(d_{\text {meta }}=0.16\right)$. These findings held true for past experiences $\left(d_{\text {meta }}\right.$ $=0.24)$ but not for those in the future $\left(d_{\text {meta }}=0.02\right)$.

3. Abstraction index

No significant truth-lie differences emerged in the abstraction index score for any individual dataset. There was, however, a significant meta effect for future experiences suggesting that truthful statements about future events are more abstract than deceptive ones $\left(d_{\text {meta }}=0.21\right)$.

\section{Named entities}

There were significant truth-lie differences in the proportion of named entities for positive $(d=0.28)$ and negative $(d=0.43)$ hotel reviews (note that this is identical to Kleinberg, Mozes, et al., 2017) as well as - in reversed

\footnotetext{
${ }^{1}$ We chose for random effects because each study was conducted in a slightly different context and the analysis, therefore, did not permit us to assume that there is one true effect size underlying all studies.
} 
magnitude - for the past weekend dataset $(d=-0.45)$. There was a significant, negative meta effect for future experiences $\left(d_{\text {meta }}=-0.20\right)$ which indicated that deceptive statements on future activities contain more named entities than truthful ones. The contradictory signs of the $d$ s of the past datasets resulted in a nonsignificant $d_{\text {meta }}$ for past events (see Discussion).

\section{Reality Monitoring (RM) score}

We found a significantly higher RM score for truthful than for deceptive reviews, both in positive $(d=0.17)$ and negative hotel reviews $(d=0.24)$. We found a positive meta-effect for past experiences $\left(d_{\text {meta }}=0.16\right)$ and a negative meta-effect for future experiences $\left(d_{\text {meta }}=-0.27\right)$ suggesting that deceptive statements have a higher RM score than truthful ones when they relate to future experiences, and vice versa for past experiences.

\section{Burgoon-Zhou specificity}

The index score of the Burgoon-Zhou statement specificity score showed significant truth-lie differences for positive $(d=-0.54)$ and negative hotel reviews $(d=-0.39)$, and for past weekend activities $(d=-0.35)$. No significant differences were observed for the future activities datasets. There was an overall meta-effect of $d_{\text {meta }}=$ -0.32 , driven by the meta effect for past experiences $\left(d_{\text {meta }}=-0.48\right)$. Deceptive statements scored higher on the statement specificity score than truthful ones.

\section{Person reference index}

The proposed person reference index showed moderate, positive effect sizes for the hotel review datasets $(d=$ 0.65 and $d=0.50$ for pos. and neg. hotel reviews, respectively), and moderate to large negative effect sizes for the past and future weekend activities datasets $(d=-0.47$ to $d=-1.28)$. There were no significant meta-analytical findings (see Table 3 \& Discussion).

\section{Motion verbs}

The proportion of motion verbs was higher in deceptive than in truthful statements for the positive hotel reviews ( $d=-0.23)$, for past weekend activities $(d=-0.33)$, and for one of the planned weekend activities datasets $(d=$ 1.01). These findings are in line with the hypothesis that deceptive language is simpler than truthful language. The meta-analytical findings lend support to that prediction for the combined datasets $\left(d_{\text {meta }}=-0.28\right)$ and statements about past experiences $\left(d_{\text {meta }}=-0.19\right)$ but not for future experiences. 
Table 3. Effect sizes per operationalization for each dataset and meta-analytical findings

\begin{tabular}{|c|c|c|c|c|c|c|c|c|c|}
\hline & \multicolumn{3}{|c|}{ Past experiences } & \multicolumn{2}{|c|}{ Future experiences } & \multirow[b]{2}{*}{$\begin{array}{c}\text { Planned } \\
\text { activities } \\
\text { III }\end{array}$} & \multicolumn{3}{|c|}{ Meta-analysis } \\
\hline & $\begin{array}{l}\text { Positive } \\
\text { hotel } \\
\text { reviews }\end{array}$ & $\begin{array}{c}\text { Negative } \\
\text { hotel } \\
\text { reviews }\end{array}$ & $\begin{array}{c}\text { Past } \\
\text { activities }\end{array}$ & $\begin{array}{c}\text { Planned } \\
\text { activities I }\end{array}$ & $\begin{array}{c}\text { Planned } \\
\text { activities } \\
\text { II }\end{array}$ & & Combined & Past & Future \\
\hline Judged & $0.45 * * *$ & $0.52 * * *$ & $0.49 * *$ & $0.23[-$ & $0.11[-$ & $0.10[-$ & $0.33 * * *$ & $0.48 * * *$ & $0.14[-$ \\
\hline Concreteness & $\begin{array}{l}{[0.31} \\
0.59]\end{array}$ & $\begin{array}{l}{[0.38} \\
0.66]\end{array}$ & $\begin{array}{l}{[0.16 ;} \\
0.82]\end{array}$ & $\begin{array}{l}0.07 \\
0.53]\end{array}$ & $\begin{array}{l}0.16 \\
0.39]\end{array}$ & $\begin{array}{l}0.17 \\
0.46]\end{array}$ & $\begin{array}{l}{[0.18} \\
0.50]\end{array}$ & $\begin{array}{l}{[0.39} \\
0.58]\end{array}$ & $\begin{array}{l}0.02 \\
0.30]\end{array}$ \\
\hline Judged & $0.47 * * *$ & $0.62 * * *$ & $0.40 *$ & $0.39 *$ & $0.40 * *$ & $0.07[-$ & $0.41 * * *$ & $0.52 * * *$ & $0.28 *$ \\
\hline $\begin{array}{l}\text { Concreteness } \\
\text { (stemmed) }\end{array}$ & $\begin{array}{l}{[0.33} \\
0.61]\end{array}$ & $\begin{array}{l}{[0.48} \\
0.76]\end{array}$ & $\begin{array}{l}{[0.07 ;} \\
0.73]\end{array}$ & $\begin{array}{l}{[0.08 ;} \\
0.69]\end{array}$ & $\begin{array}{l}{[0.13 ;} \\
0.68]\end{array}$ & $\begin{array}{l}0.19 ; \\
0.34]\end{array}$ & $\begin{array}{l}{[0.26} \\
0.57]\end{array}$ & $\begin{array}{l}{[0.40} \\
0.65]\end{array}$ & $\begin{array}{l}{[0.06} \\
0.50]\end{array}$ \\
\hline$L C M$ & $\begin{array}{c}\mathbf{0 . 2 7} * * * \\
{[0.13} \\
0.41]\end{array}$ & $\begin{array}{c}\mathbf{0 . 1 9} * * \\
{[0.06 ;} \\
0.33]\end{array}$ & $\begin{array}{c}0.28[- \\
0.05 ; \\
0.60]\end{array}$ & $\begin{array}{c}0.08[- \\
0.22 \\
0.38]\end{array}$ & $\begin{array}{c}0.20[- \\
0.07 \\
0.48]\end{array}$ & $\begin{array}{c}-0.06[- \\
0.33 \\
0.20]\end{array}$ & $\begin{array}{c}\mathbf{0 . 1 6} \text { ** } \\
{[0.05 ;} \\
0.27]\end{array}$ & $\begin{array}{c}\mathbf{0 . 2 4} * * * \\
{[0.14} \\
0.33]\end{array}$ & $\begin{array}{c}0.02[- \\
0.16 \\
0.20]\end{array}$ \\
\hline Abstraction $* 100$ & $\begin{array}{c}-0.11[- \\
0.25 \\
0.03]\end{array}$ & $\begin{array}{c}0.10[- \\
0.04 \\
0.24]\end{array}$ & $\begin{array}{c}-0.02[- \\
0.34 \\
0.31]\end{array}$ & $\begin{array}{c}0.27[- \\
0.04 \\
0.57]\end{array}$ & $\begin{array}{c}0.16[- \\
0.11 ; \\
0.44]\end{array}$ & $\begin{array}{c}0.20[- \\
0.07 \\
0.46]\end{array}$ & $\begin{array}{c}0.08[- \\
0.05 ; \\
0.20]\end{array}$ & $\begin{array}{c}-0.01[- \\
0.16 \\
0.15]\end{array}$ & $\begin{array}{c}\mathbf{0 . 2 1} \\
{[0.04} \\
0.37]\end{array}$ \\
\hline $\begin{array}{l}\text { Named } \\
\text { entities } * 100\end{array}$ & $\begin{array}{c}\mathbf{0 . 2 8}^{* * *} \\
{[0.14} \\
0.42]\end{array}$ & $\begin{array}{c}\mathbf{0 . 4 3} * * * \\
{[0.29} \\
0.57]\end{array}$ & $\begin{array}{c}\mathbf{- 0 . 4 5}{ }^{* *}[- \\
0.78 ;- \\
0.12]\end{array}$ & $\begin{array}{c}-0.11[- \\
0.40 \\
0.19]\end{array}$ & $\begin{array}{c}-0.21[- \\
0.49 \\
0.06]\end{array}$ & $\begin{array}{c}-0.26[- \\
0.52 \\
0.01]\end{array}$ & $\begin{array}{c}-0.03[- \\
0.31 \\
0.25]\end{array}$ & $\begin{array}{c}0.11[- \\
0.41 ; \\
0.62]\end{array}$ & $\begin{array}{c}\mathbf{- 0 . 2 0} *[- \\
0.361 \\
0.04]\end{array}$ \\
\hline$R M$ & $\begin{array}{c}\mathbf{0 . 1 7} * \\
{[0.04 ;} \\
0.31]\end{array}$ & $\begin{array}{c}\mathbf{0 . 2 4} * * * \\
{[0.10} \\
0.38]\end{array}$ & $\begin{array}{c}-0.11[- \\
0.44 \\
0.21]\end{array}$ & $\begin{array}{c}-0.23[- \\
0.53 \\
0.07]\end{array}$ & $\begin{array}{c}\mathbf{- 0 . 4 2} * *[- \\
0.70 ;- \\
0.15]\end{array}$ & $\begin{array}{c}-0.15[- \\
0.41 \\
0.12]\end{array}$ & $\begin{array}{c}-0.07[- \\
0.27 \\
0.15]\end{array}$ & $\begin{array}{c}\mathbf{0 . 1 6} * * \\
{[0.05} \\
0.29]\end{array}$ & $\begin{array}{c}-\mathbf{0 . 2 7} * * \\
0.43 ;- \\
0.10]\end{array}$ \\
\hline Burgoon-Zhou & $\begin{array}{c}\mathbf{- 0 . 5 5} \text { *** } \\
{[-0.69 ;-} \\
0.41]\end{array}$ & $\begin{array}{c}\mathbf{- 0 . 4 4} * * * \\
{[-0.58 ;-} \\
0.30]\end{array}$ & $\begin{array}{c}\mathbf{- 0 . 3 5} *[- \\
0.68 ;- \\
0.03]\end{array}$ & $\begin{array}{c}-0.28[- \\
0.58 \\
0.02]\end{array}$ & $\begin{array}{c}-0.19[- \\
0.46 \\
0.09]\end{array}$ & $\begin{array}{l}0.01[- \\
0.25 ; \\
0.27]\end{array}$ & $\begin{array}{c}\mathbf{- 0 . 3 2} * *[- \\
-0.49 ;- \\
0.15]\end{array}$ & $\begin{array}{c}\mathbf{- 0 . 4 8} * * * \\
{[-0.58 ;-} \\
0.39]\end{array}$ & $\begin{array}{c}-0.14[- \\
0.31 \\
0.03]\end{array}$ \\
\hline Person index & $\begin{array}{c}\mathbf{0 . 6 5} * * * \\
{[0.50} \\
0.79]\end{array}$ & $\begin{array}{c}\mathbf{0 . 5 0} * * * \\
{[0.36} \\
0.64]\end{array}$ & $\begin{array}{c}\mathbf{- 1 . 2 8} * * * \\
{[-1.63 ;-} \\
0.92]\end{array}$ & $\begin{array}{c}\mathbf{- 1 . 0 0} * * * \\
{[-1.31 ;-} \\
0.68]\end{array}$ & $\begin{array}{c}\mathbf{- 0 . 4 7} * *[- \\
0.74 ;- \\
0.19]\end{array}$ & $\begin{array}{c}-0.01[- \\
0.28 \\
0.25]\end{array}$ & $\begin{array}{c}-0.26[- \\
0.89 \\
0.37]\end{array}$ & $\begin{array}{c}-0.03[- \\
1.24 \\
1.17]\end{array}$ & $\begin{array}{c}-0.49[- \\
1.04 \\
0.07]\end{array}$ \\
\hline Motion & $\begin{array}{c}\mathbf{- 0 . 2 3} * *[- \\
0.37 ;- \\
0.09]\end{array}$ & $\begin{array}{c}-0.10[- \\
0.23 \\
0.04]\end{array}$ & $\begin{array}{c}-\mathbf{0 . 3 3} *[- \\
0.66 ;- \\
0.01]\end{array}$ & $\begin{array}{c}\mathbf{- 1 . 0 1} * * * \\
{[-1.33 ;-} \\
0.70]\end{array}$ & $\begin{array}{c}-0.10[- \\
0.38 \\
0.17]\end{array}$ & $\begin{array}{c}0.01[- \\
0.26 \\
0.27]\end{array}$ & $\begin{array}{c}-\mathbf{- 0 . 2 8} *[- \\
0.56 \\
0.00]\end{array}$ & $\begin{array}{c}-\mathbf{0 . 1 9} * *[- \\
0.31 \\
0.06]\end{array}$ & $\begin{array}{c}-0.36[- \\
0.99 \\
0.27]\end{array}$ \\
\hline
\end{tabular}

Note. $*=$ sign. at $p<.05 ; * *=$ sign. at $p<.01 ; * * *=$ sign. at $p<.001$.

\section{Predicting veracity}

While the above analyses examine the difference between truthful and deceptive texts in various concreteness operationalizations, another interesting aspect is that of the predictive power. To assess what the best combination of concreteness indicators is to make a truth-lie prediction, we built a generalized linear mixed-effects model (GLMM) using the binomial model for logistic regression on the binary outcome 'veracity' (truthful vs deceptive). In that model, the dataset was controlled for by adding it as a random intercept. Using all eight concreteness indices $^{2}$, we built the model on randomly selected $50 \%$ of the data and validated its predictive performance on the other $50 \%$ of the data.

Table 4 (see also Appendix B) shows that Brysbaert et al.'s judged concreteness (stemmed) and the Burgoon-Zhou specificity were consistently strong predictors of veracity ${ }^{3}$. There were temporally moderated

\footnotetext{
${ }^{2}$ We used the judged concreteness stemmed version only (and not judged concreteness).

${ }^{3}$ The abstraction index $(\beta=-0.14, z=-2.07, p=.039)$ was marginally significant.
} 
effects for the person index (past), motion verbs (future) and the abstraction index (past and future). Transforming the log-odds coefficients implies that positive $\beta$-coefficients mean that an increase in the predictor, increases the odds of texts being deceptive (and vice versa for being truthful). The prediction results showed that the accuracy was significantly above the chance level for the combined datasets $(62.65 \%)$ as well as for both temporal foci separately (past: 64.64\%; future: 61.54\%). As expected the classification accuracies were slightly higher in the model building phase but remained relatively stable when tested in the model validation phase.

Table 4. $\beta$-coefficients (non-transformed) of the logistic regression GLMM.

\begin{tabular}{lccc}
\hline \multicolumn{1}{c}{ Predictor } & All datasets & Past activities & Planned activities \\
\hline Concreteness stemmed & $-0.46^{* * *}$ & $-0.60^{* * *}$ & $-0.50^{* * *}$ \\
LCM & 0.04 & 0.11 & 0.03 \\
Named entities & 0.09 & -0.02 & $0.27^{*}$ \\
RM score & 0.00 & -0.02 & 0.32 \\
Person index & -0.06 & $-0.42^{* * *}$ & $0.66^{* * *}$ \\
Burgoon-Zhou & $0.41^{* * *}$ & $0.47 * * *$ & $0.63^{* * *}$ \\
Motion verbs & 0.21 & 0.11 & $0.39^{* * *}$ \\
Abstraction index & $-0.19 *$ & -0.20 & $-0.63 * * *$ \\
Intercept (dataset) & -0.04 & 0.06 & 0.18 \\
& & & -176.3 \\
Log-Likelihood model fit & -759.2 & -529.9 & $68.54 * * *[62.74 ; 73.56]$ \\
Accuracy (model-building phase) & $63.11^{* * *}[60.27 ; 65.88]$ & $67.74 * * *[64.52 ; 70.84]$ & $61.54 * *[55.76 ; 67.08]$ \\
Accuracy (model validation phase) & $62.65^{* * *}[59.81 ; 65.43]$ & $64.64 * * *[61.36 ; 67.82]$ & 6.06 \\
\hline
\end{tabular}

Note. $*=$ sign. at $p<.05 ; * * *=$ sign. at $p<.001$. For accuracies the $p$-value represents refers to the binomial test against the chance level.

\section{Discussion}

The present investigation explored the concept of linguistic concreteness for deception detection in six datasets. We used eight operationalizations from the literature to represent concreteness in a computer-automated manner and tested two competing hypotheses. i) The truthful concreteness hypothesis suggested that truth-tellers' language would be more concrete than that of liars because the latter fail to recall an event in sufficient detail from memory and are reluctant to provide potentially testable information. ii) The deceptive concreteness hypothesis predicted that liars' language would be more concrete because cognitive depletion limits the use of rich and evaluative language, making liars resort to concrete, simple language. Moreover, liars are thought to be inclined to appear convincing and attempt to do so by including specific, concrete information into their narratives.

Are truth tellers or liars more concrete?

In line with the truthful concreteness hypothesis, the direct operationalization of concreteness based on Brysbaert et al. (2014) showed that truthful statements contained more concrete language than deceptive statements. Zooming in more closely, deceptive hotel reviews had a lower judged concreteness score, a lower Reality Monitoring score, a lower proportion of named entities, and a lower score on the person reference index 
(see Table 5). These findings lend support to the truthful concreteness hypothesis for two hotel review datasets of different valence (positive and negative), characterizing deceptive language by a lack of specific information and concreteness. This may be viewed as in line with the Reality Monitoring, CBCA, VA, and CLT approaches to deception detection, in that truthful narratives are concrete, contextually embedded and rich in detail, whereas deceptive narratives lack such information. For one of the planned activities datasets (future weekend plans I), we found two strong effects: deceptive statements contained more motion verbs and a higher person reference index. All future weekend plans of truth-tellers obtained a higher concreteness score than liars; this finding also received meta-analytical support. However, albeit less consistent, the deceptive concreteness hypothesis also received partial support: The mini meta-analysis showed that deceptive future experiences seemed to have a lower abstraction score (i.e., they are less abstract, hence more concrete), contain more named entities and a higher RM score than truthful intentions, all pointing to more concrete language in deceptive statements.

Table 5. Summary of meta-analytical findings

\begin{tabular}{|c|c|c|c|c|}
\hline Operationalization & Past & Future & Combined & In line with the hypothesis? \\
\hline Concreteness & + & + & + & Support for the truthful concreteness hypothesis \\
\hline Linguistic Category & + & 0 & + & Partial support for the deceptive concreteness hypothesis \\
\hline \multicolumn{5}{|l|}{ Model } \\
\hline Abstraction index & 0 & + & 0 & Weak support for the truthful concreteness hypothesis \\
\hline Named entities & 0 & - & 0 & Inconsistent with the deceptive concreteness hypothesis \\
\hline Reality Monitoring & + & - & 0 & Partial support for the truthful concreteness hypothesis (past) \\
\hline score & & & & $\begin{array}{l}\text { Partial support for the deceptive concreteness hypothesis } \\
\text { (future) }\end{array}$ \\
\hline Linguistic specificity & - & 0 & - & Partial support for the deceptive concreteness hypothesis \\
\hline Person index & 0 & 0 & 0 & - \\
\hline Motion verbs & - & 0 & - & Partial support for the deceptive concreteness hypothesis \\
\hline
\end{tabular}

Note. A (+) sign indicates that this operationalization was significantly larger in truth-tellers than in liars. A (-) indicates the opposite. If there was no difference, this is indicated by a 0 .

Rather than showing a consistent pattern of the role of linguistic concreteness for deception, the current findings shed light on three potential dynamics. First, the results suggest that the current operationalizations might capture more than just one overarching concept of concreteness. If all operationalizations were representations of concreteness, we would have expected to see a coherent pattern across the different operationalizations (i.e., the same directionality of effects within each dataset). For example, most concreteness indices used in the present investigation show that for hotel reviews, truthful language is more concrete than deceptive language. However, the stark outlier is the Burgoon-Zhou concept of specificity: we find support for an opposite pattern for this measure of concreteness. Exploratory analyses on the positive hotel reviews dataset $(d=-0.55)$ showed that the 
main drivers of that effect were first-person singular pronouns ${ }^{4}(d=-0.83[-0.97 ;-0.68])$, which were more prevalent in deceptive than in truthful reviews. While this might emphasize the important role of pronouns for linguistic deception (Newman et al., 2003), it is also possible that the adoption of the first-person perspective is due to the context of hotel reviews that are inherently about the subjective experience of a hotel guest. This finding might add to the idea that liars have trouble, not only in producing richer language but in taking on the truthtellers' perspective: they are not aware of the language that a truth-teller would use. It would be interesting to see whether these results are generalizable to settings where both the liars and truth-tellers have been to the hotel. In general, the current findings show that it is possible that not all operationalizations measure the construct of concreteness and that not all operationalizations are equally suitable for deception detection.

Second, the divergent results obtained across datasets suggest that concreteness as a cue to deception may be context-dependent. The hotel review datasets showed most results in the expected direction and findings were consistent between positive and negative hotel reviews, while little to no such effects were evident for the other datasets. This finding highlights that the concept of concreteness as a cue to deception might indeed be dependent on the functional setting of a communication act (e.g. reviewing versus describing activities). Moreover, the person reference index highlights the role of temporal focus of deceptive language. The observed effects for the abstraction index and the named entities held true for statements about future events but not for past experiences. Simple language as measured through motion verbs, and the Burgoon-Zhou specificity were primarily driven by past experiences, while the Reality Monitoring index was expectedly higher in truths than in lies on past events but was reversed for future events. Some findings for the person reference index are in line with the truthful concreteness hypothesis (i.e., for the hotel reviews, truthful $>$ deceptive), yet they support the deceptive concreteness hypothesis in the past activities data and for two planned activities datasets. This, too, highlights the role of the context since a clear temporal explanation (e.g., that lies about future events are overcompensated, and that lies about past events are less concrete) is not supported.

Third, it is possible that concreteness behaves dynamically within statements. Such dynamics could entail (i) that liars (truth-tellers) are highly specific on some aspects of their statement at the cost of other aspects, and (ii) that concreteness varies over the narrative time. The latter means that it is possible that a narrator uses concrete language at the start of the statement but resorts to vaguer language nearing the end of the statement. Such dynamics are blurred (i.e., they cancel each other out) by concreteness point-estimates and it needs future research looking at intra-textual dynamics of language use for deception detection.

Classification analysis showed that concreteness dimensions are useful in discriminating truthful from deceptive statements with modest above chance level accuracy. That the accuracies in predictive analysis remained stable in an independent model validation phase might hint at the generalizability, possibly due to deriving theoretically-motivated cues to deception. The obtained accuracies ranging between $61.18 \%$ and $64.64 \%$ are in line with the majority of verbal and linguistic deception detection (e.g., Masip et al., 2005; Mihalcea \& Strapparava, 2009; Pérez-Rosas \& Mihalcea, 2014; Vrij, Fisher, \& Blank, 2017) and show that computer-

\footnotetext{
${ }^{4}$ The other sub-indices in ascending order of effect size: relative perceptual details $(d=-0.22[-0.36 ;-0.08])$, adverbs $(d=$ $0.20[-0.33 ;-0.06])$, third-person pronouns plural $(d=-0.21,[-0.35 ; 0.08])$, second-person pronouns $(d=-0.17[-0.30$; $0.03])$, adjectives $(d=-0.07, n s,[-0.21 ; 0.07])$, quantity of perceptual details $(d=-0.06, n s,[-0.20 ; 0.08])$, third-person pronouns singular $(d=-0.02, n s,[-0.16 ; 0.12])$, and first-person plural pronouns $(d=0.24[0.10 ; 0.38])$.
} 
automated verbal deception detection can be used to as part of a broader classification procedure with abovechance accuracy.

\section{Outlook}

The current investigation suggests that future research is needed to disentangle the multifaceted linguistic dimensions that might underlie the concept of concreteness. Multiple theories provide a guiding framework that points to a critical role of concrete language in deception, and it would, therefore, be interesting to specify further the linguistic constructs that can test and falsify concreteness predictions. Likewise, the divergent findings presented here suggest that it would be beneficial to map out the boundary conditions of different linguistic constructs for deception detection to gain a better understanding of what works when. Other suggestions for future research are conducting more multi-dataset and multi-operationalization investigations and looking at intratextual patterns of concreteness and other linguistic constructs. The latter will allow us to examine whether liars and truth-tellers employ different strategies in language use throughout their statements - an aspect that is currently overlooked in deception research. The present study showed that different operationalizations result in different effects even within one dataset and that different datasets yield different effects for single operationalizations. Utilizing the concreteness indices hinted at better than chance classification accuracy, suggesting that concreteness is a linguistic concept worth exploring further. In the absence of consistent truth-lie differences, single studies on deceptive language seem to provide a skewed picture of the field.

\section{Conclusion}

This study provided a comprehensive analysis of linguistic concreteness for verbal deception research. The findings are context- and operationalization-dependent and show that only by using multiple datasets and operationalizations, a broader overview of the field can be obtained. Concreteness is a diverse linguistic concept, and it seems worthwhile to examine its relation to deception in the future. 


\section{Appendix A.}

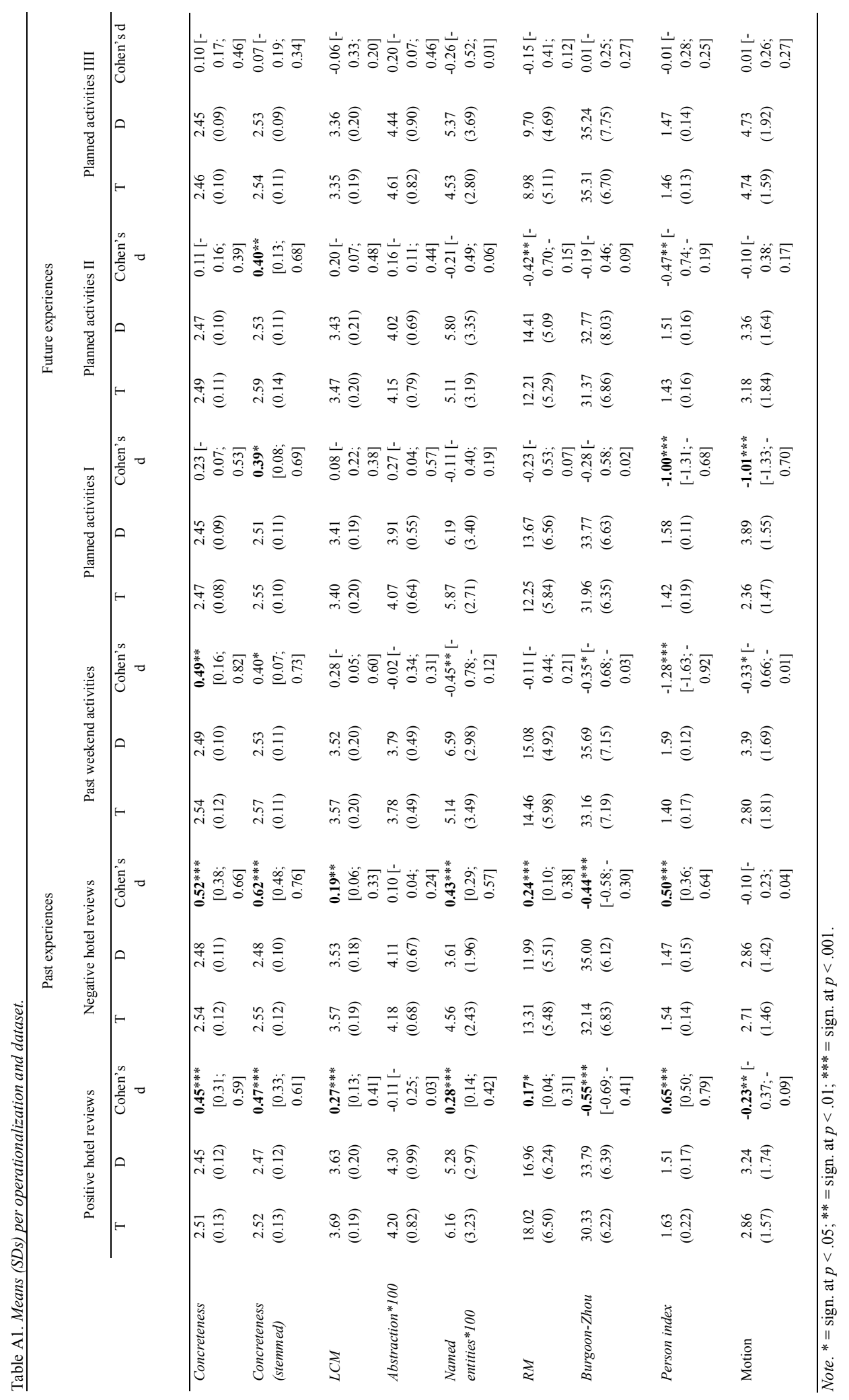




\section{Appendix B.}

Table B1. Full model results of the GLMM logistic regression in the model building phase (all datasets)

\begin{tabular}{lcccc}
\hline \multicolumn{1}{c}{ Predictor } & $\begin{array}{c}\text { Coefficient } \boldsymbol{\beta} \\
\text { estimate (non- } \\
\text { transformed) }\end{array}$ & Standard error of $\boldsymbol{\beta}$ & z-value & p-value \\
\hline Concreteness & -0.41 & 0.06 & -6.46 & $<.001$ \\
stemmed & -0.00 & 0.06 & -0.05 & .959 \\
LCM & -0.01 & 0.07 & -0.09 & .931 \\
Named entities & 0.06 & 0.06 & 0.86 & .390 \\
RM score & -0.10 & 0.06 & -1.56 & .119 \\
Person index & 0.39 & 0.07 & 5.55 & $<.001$ \\
Burgoon-Zhou & 0.23 & 0.06 & 3.64 & $<.001$ \\
Motion verbs & -0.14 & 0.07 & -2.07 & .039 \\
Abstraction index & -0.01 & 0.06 & -0.23 & .821 \\
Intercept (dataset) & & & & \\
\hline
\end{tabular}

Table B2. Full model results of the GLMM logistic regression in the model building phase (past)

\begin{tabular}{lcccc}
\hline \multicolumn{1}{c}{ Predictor } & $\begin{array}{c}\text { Coefficient } \boldsymbol{\beta} \\
\text { estimate (non- } \\
\text { transformed) }\end{array}$ & Standard error of $\boldsymbol{\beta}$ & z-value & p-value \\
\hline Concreteness & -0.60 & 0.09 & -6.83 & $<.001$ \\
stemmed & & & & .211 \\
LCM & 0.11 & 0.09 & 1.25 & .816 \\
Named entities & -0.02 & 0.09 & -0.22 & .781 \\
RM score & -0.02 & 0.09 & -0.28 & $<.001$ \\
Person index & -0.42 & 0.09 & -4.81 & $<.001$ \\
Burgoon-Zhou & 0.47 & 0.10 & 4.93 & .201 \\
Motion verbs & 0.11 & 0.09 & 1.28 & .146 \\
Abstraction index & -0.20 & 0.14 & -1.45 & .447 \\
Intercept (dataset) & 0.06 & 0.08 & 0.76 & \\
\hline
\end{tabular}

Table B3. Full model results of the GLMM logistic regression in the model building phase (future)

\begin{tabular}{lcccc}
\hline \multicolumn{1}{c}{ Predictor } & $\begin{array}{c}\text { Coefficient } \boldsymbol{\beta} \\
\text { estimate (non- } \\
\text { transformed) }\end{array}$ & Standard error of $\boldsymbol{\beta}$ & z-value & p-value \\
\hline Concreteness & -0.50 & 0.17 & -6.83 & .003 \\
stemmed & 0.03 & 0.16 & 0.18 & .856 \\
LCM & 0.27 & 0.13 & 2.12 & .034 \\
Named entities & 0.32 & 0.17 & 1.86 & .062 \\
RM score & 0.66 & 0.17 & 3.96 & $<.001$ \\
Person index & 0.63 & 0.18 & 3.57 & $<.001$ \\
Burgoon-Zhou & 0.39 & 0.13 & 2.99 & .001 \\
Motion verbs & -0.63 & 0.28 & -2.24 & .025 \\
Abstraction index & 0.18 & 0.19 & 0.93 & .447 \\
Intercept (dataset) & & & & \\
\hline
\end{tabular}




\section{References}

Benoit, K., \& Matsuo, A. (2017). Spacyr: R wrapper to the spacy NLP library (Version R package version 0.9.0). Retrieved from https://CRAN.R-project.org/package=spacyr

Bond, G. D., Holman, R. D., Eggert, J.-A. L., Speller, L. F., Garcia, O. N., Mejia, S. C., ... Rustige, R. (2017). 'Lyin”, Ted', “Crooked Hillary”, and "Deceptive Donald”: Language of lies in the 2016 US presidential debates: Language of lies in debates.' Applied Cognitive Psychology, 31(6), 668-677. https://doi.org/10.1002/acp.3376

Bond, G. D., \& Lee, A. Y. (2005). Language of lies in prison: Linguistic classification of prisoners' truthful and deceptive natural language. Applied Cognitive Psychology, 19(3), 313-329.

Brysbaert, M., Warriner, A. B., \& Kuperman, V. (2014). Concreteness ratings for 40 thousand generally known English word lemmas. Behavior Research Methods, 46(3), 904-911. https://doi.org/10.3758/s13428013-0403-5

Buller, D. B., Burgoon, J. K., Buslig, A. L. S., \& Roiger, J. F. (1994). Interpersonal Deception VIII: Further Analysis of Nonverbal and Verbal Correlates of Equivocation from the Bavelas et al. (1990) Research. Journal of Language and Social Psychology, 13(4), 396-417. https://doi.org/10.1177/0261927X94134003

Buller, D. B., Burgoon, J. K., Buslig, A., \& Roiger, J. (1996). Testing Interpersonal Deception Theory: The Language of Interpersonal Deception. Communication Theory, 6(3), 268-289. https://doi.org/10.1111/j.1468-2885.1996.tb00129.x

Burgoon, J. K., \& Qin, T. (2006). The dynamic nature of deceptive verbal communication. Journal of Language and Social Psychology, 25(1), 76-96. https://doi.org/10.1177/0261927X05284482

Burleson, B. R. (2009). Understanding the outcomes of supportive communication: A dual-process approach. Journal of Social and Personal Relationships, 26(1), 21-38. https://doi.org/10.1177/0265407509105519

Cohen, J. (1988). Statistical power analysis for the behavioral sciences. New York, NY: Academic Press.

Fuller, C. M. (2008). High-stakes, real-world deception: An examination of the process of deception and deception detection using linguistic-based cues. Oklahoma State University.

Geurts, R., Granhag, P. A., Ask, K., \& Vrij, A. (2016). Taking threats to the lab: Introducing an experimental paradigm for studying verbal threats. Journal of Threat Assessment and Management, 3(1), 53-64. https://doi.org/10.1037/tam0000060 
Gollwitzer, P. M. (1999). Implementation intentions: Strong effects of simple plans. American Psychologist, 54(7), 493-503. https://doi.org/10.1037/0003-066X.54.7.493

Granhag, P. A., \& Mac Giolla, E. (2014). Preventing future crimes: Identifying markers of true and false intent. European Psychologist, 19(3), 195-206. https://doi.org/10.1027/1016-9040/a000202

Harvey, A. C., Vrij, A., Leal, S., Lafferty, M., \& Nahari, G. (2017). Insurance based lie detection: Enhancing the verifiability approach with a model statement component. Acta Psychologica, 174, 1-8. https://doi.org/10.1016/j.actpsy.2017.01.001

Hauch, V., Blandón-Gitlin, I., Masip, J., \& Sporer, S. L. (2015). Are computers effective lie detectors? A metaanalysis of linguistic cues to deception. Personality and Social Psychology Review, 19(4), 307-342. https://doi.org/10.1177/1088868314556539

Honnibal, M. (2017). Spacy (Version 1.9.0). Retrieved from https://spacy.io/

Johnson, M. K., Bush, J. G., \& Mitchell, K. J. (1998). Interpersonal reality monitoring: Judging the sources of other people's memories. Social Cognition, 16(2), 199-224. https://doi.org/10.1521/soco.1998.16.2.199

Jupe, L. M., Leal, S., Vrij, A., \& Nahari, G. (2017). Applying the Verifiability Approach in an international airport setting. Psychology, Crime \& Law, 23(8), 812-825. https://doi.org/10.1080/1068316X.2017.1327584

Kleinberg, B., Mozes, M., Arntz, A., \& Verschuere, B. (2017). Using named entities for computer-automated verbal deception detection. Journal of Forensic Sciences, 63(3), 714-723. https://doi.org/10.1111/1556-4029.13645

Kleinberg, B., Nahari, G., Arntz, A., \& Verschuere, B. (2017). An investigation on the detectability of deceptive intent about flying through verbal deception detection. Collabra: Psychology, 3(1), 21. https://doi.org/10.1525/collabra. 80

Kleinberg, B., Toolen, Y. van der, Vrij, A., Arntz, A., \& Verschuere, B. (2018). Automated verbal credibility assessment of intentions: The model statement technique and predictive modeling. Applied Cognitive Psychology, 32(3), 354-366. https://doi.org/10.1002/acp.3407

Köhnken, G. (2004). Statement validity analysis and the 'detection of the truth.' The Detection of Deception in Forensic Contexts, 41-63. 
Larrimore, L., Jiang, L., Larrimore, J., Markowitz, D., \& Gorski, S. (2011). Peer to Peer Lending: The Relationship Between Language Features, Trustworthiness, and Persuasion Success. Journal of Applied Communication Research, 39(1), 19-37. https://doi.org/10.1080/00909882.2010.536844

Liberman, N., \& Trope, Y. (1998). The role of feasibility and desirability considerations in near and distant future decisions: A test of temporal construal theory. Journal of Personality and Social Psychology, 75(1), 5-18. https://doi.org/10.1037/0022-3514.75.1.5

Markowitz, D. M., \& Hancock, J. T. (2016). Linguistic obfuscation in fraudulent science. Journal of Language and Social Psychology, 35(4), 435-445. https://doi.org/10.1177/0261927X15614605

Masip, J., Sporer, S. L., Garrido, E., \& Herrero, C. (2005). The detection of deception with the reality monitoring approach: a review of the empirical evidence. Psychology, Crime \& Law, 11(1), 99-122. https://doi.org/10.1080/10683160410001726356

Mihalcea, R., \& Strapparava, C. (2009). The lie detector: Explorations in the automatic recognition of deceptive language. In Proceedings of the ACL-IJCNLP 2009 Conference Short Papers (pp. 309-312). Association for Computational Linguistics. Retrieved from http://dl.acm.org/citation.cfm?id=1667679

Nahari, G., Vrij, A., \& Fisher, R. P. (2014). Exploiting liars' verbal strategies by examining the verifiability of details. Legal and Criminological Psychology, 19(2), 227-239. https://doi.org/10.1111/j.20448333.2012.02069.x

Newman, M. L., Pennebaker, J. W., Berry, D. S., \& Richards, J. M. (2003). Lying words: Predicting deception from linguistic styles. Personality and Social Psychology Bulletin, 29(5), 665-675. https://doi.org/10.1177/0146167203029005010

Ott, M., Cardie, C., \& Hancock, J. T. (2013). Negative deceptive opinion spam. In HLT-NAACL (pp. 497-501). Retrieved from http://www.aclweb.org/website/old_anthology/N/N13/N13-1.pdf\#page=535

Ott, M., Choi, Y., Cardie, C., \& Hancock, J. T. (2011). Finding deceptive opinion spam by any stretch of the imagination. In Proceedings of the 49th Annual Meeting of the Association for Computational Linguistics: Human Language Technologies-Volume 1 (pp. 309-319). Association for Computational Linguistics. Retrieved from http://dl.acm.org/citation.cfm?id=2002512

Pennebaker, J. W., Boyd, R. L., Jordan, K., \& Blackburn, K. (2015). The development and psychometric properties of LIWC2015. Retrieved from https://repositories.lib.utexas.edu/handle/2152/31333

Pérez-Rosas, V., \& Mihalcea, R. (2014). Cross-cultural deception detection. In ACL (2) (pp. 440-445). Retrieved from http://www.anthology.aclweb.org/P/P14/P14-2072.pdf 
R Core Team. (2016). R: A language and environment for statistical computing. Vienna, Austria: R Foundation for Statistical Computing. Retrieved from https://www.R-project.org/

Seih, Y.-T., Beier, S., \& Pennebaker, J. W. (2017). Development and examination of the Linguistic Category Model in a computerized text analysis method. Journal of Language and Social Psychology, 36(3), 343-355. https://doi.org/10.1177/0261927X16657855

Semin, G. R., \& Fiedler, K. (1988). The cognitive functions of linguistic categories in describing persons: Social cognition and language. Journal of Personality and Social Psychology, 54(4), 558-568. https://doi.org/10.1037/0022-3514.54.4.558

Tiantian Qin, Burgoon, J. K., Blair, J. P., \& Nunamaker, J. F. (2005). Modality Effects in Deception Detection and Applications in Automatic-Deception-Detection (pp. 23b-23b). IEEE. https://doi.org/10.1109/HICSS.2005.436

Trope, Y., \& Liberman, N. (2010). Construal-level theory of psychological distance. Psychological Review, 117(2), 440-463. https://doi.org/10.1037/a0018963

Undeutsch, U. (1989). The development of statement reality analysis. In Credibility assessment (pp. 101-119). Springer. Retrieved from http://link.springer.com/chapter/10.1007/978-94-015-7856-1_6

Valentine, J. C., Pigott, T. D., \& Rothstein, H. R. (2010). How many studies do you need?: A primer on statistical power for meta-analysis. Journal of Educational and Behavioral Statistics, 35(2), 215-247. https://doi.org/10.3102/1076998609346961

Vallacher, R. R., \& Wegner, D. M. (1987). What do people think they're doing? Action identification and human behavior. Psychological Review, 94(1), 3-15. https://doi.org/10.1037/0033-295X.94.1.3

Viechtbauer, W. (2010). Conducting meta-analyses in R with the metafor package. Journal of Statistical Software, 36(3). https://doi.org/10.18637/jss.v036.i03

Vrij, A., Fisher, R. P., \& Blank, H. (2017). A cognitive approach to lie detection: A meta-analysis. Legal and Criminological Psychology, 22(1), 1-21. https://doi.org/10.1111/lcrp.12088

Zhou, L., Burgoon, J. K., Nunamaker, J. F., \& Twitchell, D. (2004). Automating linguistics-based cues for detecting deception in text-based asynchronous computer-mediated communications. Group Decision and Negotiation, 13(1), 81-106. https://doi.org/10.1023/B:GRUP.0000011944.62889.6f

Zuckerman, M., DePaulo, B. M., \& Rosenthal, R. (1981). Verbal and nonverbal communication of deception. Advances in Experimental Social Psychology, 14, 1-59. https://doi.org/10.1016/S0065-2601(08)60369$\mathrm{X}$ 
